\title{
PDE5 Overexpression in Well-Differentiated Thyroid Carcinomas Is Associated with Lymph Node Metastasis
}

\author{
Ning Zhang, ${ }^{1}$ Zeng Fang, ${ }^{1,2}$ Qiufang $\mathrm{Li}^{2}{ }^{2}$ Kebing Wang, ${ }^{2}$ Songqi $\mathrm{Li}^{1}{ }^{1}$ Wen $\mathrm{Li}^{2}{ }^{2}$ and \\ Shenming Wang ${ }^{1,3}$ \\ ${ }^{1}$ Division of Breast and Thyroid Surgery, First Affiliated Hospital, Sun Yat-sen University, Guangzhou, China \\ ${ }^{2}$ Laboratory of General Surgery, First Affiliated Hospital, Sun Yat-sen University, Guangzhou, China \\ ${ }^{3}$ Division of Vascular Surgery, First Affiliated Hospital, Sun Yat-sen University, Guangzhou, China
}

Correspondence should be addressed to Wen Li; wenli28@163.com and Shenming Wang; shenmingwang@hotmail.com

Received 6 March 2017; Revised 24 May 2017; Accepted 31 May 2017; Published 1 October 2017

Academic Editor: Aldo E. Calogero

Copyright (c) 2017 Ning Zhang et al. This is an open access article distributed under the Creative Commons Attribution License, which permits unrestricted use, distribution, and reproduction in any medium, provided the original work is properly cited.

\begin{abstract}
Overexpression of PDE5 is observed in certain human cancers, but PDE5 expression in well-differentiated thyroid carcinoma (WDTC) is unknown. We therefore examined PDE5 expression and its relationship with the clinicopathological features of WDTC. Real-time qPCR and Western blotting were performed to analyze the expression of PDE5 mRNA and protein in paired WDTC tumor and adjacent nontumor tissues. Immunohistochemistry was used to analyze the expression of PDE5 in paraffinembedded tissues obtained from 103 cases of WDTC. Statistical analyses were performed to examine the correlation between PDE5 expression and clinicopathological features. The expression of PDE5 mRNA and protein was upregulated in WDTC lesions compared to their paired noncancerous tissues. The expression of PDE5 was significantly correlated with age $(P=0.032)$, regional lymph node status $(P=0.004)$, and the presence of distant metastasis $(P=0.020)$. High PDE5 expression was more closely associated with lymph node involvement in patients over 45 years $(\mathrm{OR}=15.60, P \leq 0.05)$. Thus, PDE5 may be a potential biomarker in WDTC, particularly in patients with regional lymph node metastasis, which is associated with disease recurrence, treatment failure, and morbidity. PDE5 expression may also help predict the prognosis and recurrence of WDTC after surgery.
\end{abstract}

\section{Introduction}

Thyroid cancer (TC) is the most common endocrine malignancy; the incidence of which has been steadily increasing over the last three decades [1]. Papillary and follicular TCs are the most common forms of TC, and they are classified as well-differentiated thyroid cancer (WDTC) based on the histological features. WDTCs are generally indolent tumors that are associated with low mortality. Although the 30-year disease-specific survival rates can exceed 95\%, the 5 -year survival is as low as $56 \%$ in patients with metastatic disease [2]. Disease recurrence or persistence is associated with high mortality and long-term treatment failure [1-4]. Regional lymph node involvement is often observed in the early stage of the disease and is associated with postoperative recurrence [5-8]. Therefore, accurate evaluation of regional lymph node status plays an important role in WDTC treatment. Fine-needle aspiration biopsy (FNAB) is a critical diagnostic test for evaluation of the nodules. However, the procedure is invasive and is associated with a misdiagnosis rate of $10-15 \%$; this limits the application of FNAB in clinical practice [3]. Therefore, it would be useful to identify other methods for evaluating the regional lymph node status in WDTC.

PDE5 is a cytoplasmic enzyme that regulates the levels of cGMP by hydrolyzing it [9]. It plays a critical role in cell growth and apoptosis [10]. Previous studies have shown that PDE5 is overexpressed in human prostate cancer, nonsmall cell lung cancer, and breast cancer [11-14]. PDE5 inhibitors have also been used for therapeutic treatment [10]. Passon and colleagues have reported that the copy number of PDE5 is amplified in papillary thyroid cancer (PTC) [15]. Further, it was reported that variation in the copy number of PDE5 contributes to tumorigenesis and the development 
of PTC [16]. Sodium iodide symporter (NIS) is associated with the differentiation of TC and postoperative ${ }^{131} \mathrm{I}$ treatment resistance [17]. Sponziello et al. found that PTC tissues with high levels of PDE5 mRNA had a lower level of NIS transcripts, which means that poor differentiation might lead to long-term treatment failure [18]. Thus, PDE5 overexpression may play a role in the cell growth and metastasis of PTC. However, the significance of PDE5 in WDTC patients is unknown. Therefore, in the present study, we examined PDE5 expression in 103 formalin-fixed, paraffin-embedded tissue specimens of human WDTC and its correlation with the clinicopathologic features of the patients.

\section{Materials and Methods}

2.1. Patients and Samples. Paraffin-embedded samples were obtained from 103 patients diagnosed with WDTC who underwent surgery between July 2009 and December 2015 at the Department of Breast and Thyroid Surgery and Department of Pathology, the First Affiliated Hospital of Sun Yat-sen University. All the patients' ages ranged from 7 to 81 years $($ median $=50)$. The patient group included 19 and 57 patients who were diagnosed with WDTC with distant metastasis and lymph node metastasis, respectively, confirmed by imaging or pathological examination, and 27 patients with primary WDTC. Clinicopathological information such as age, tumor size, lymph node status, and histological type was obtained by reviewing their medical records and pathology reports.

Four matched pairs of fresh WDTC tumor and adjacent noncancerous tissue samples (at least $2 \mathrm{~cm}$ away from the margin of the tumor tissue) were also obtained for determining the mRNA and protein levels of PDE5. Histopathological analysis with hematoxylin-eosin staining of frozen sections confirmed that the tumor tissues comprised $>70 \%$ cancer cells without necrosis and that no cancer lesions were present in the noncancerous tissues.

The study was approved by the Medical Ethical Committee of the First Affiliated Hospital of Sun Yat-sen University (Guangzhou, China). Informed consent was obtained from all the patients for the use of their clinical specimens.

2.2. RNA Extraction and Real-Time qPCR. We followed the methods of Zhang et al. [19]. Total RNA from the primary tumor and adjacent nontumor tissue samples was extracted using TRIzol reagent (Invitrogen, Carlsbad, CA, USA) according to the manufacturer's instructions. RNA concentration and quality were assessed spectrophotometrically at 260 and $280 \mathrm{~nm}$. The enzyme and reagents for reverse transcription and PCR amplification were obtained from Roche. The cDNAs were amplified by PCR $\left(42^{\circ} \mathrm{C}\right.$ for $15 \mathrm{~min}, 85^{\circ} \mathrm{C}$ for $5 \mathrm{~min}$, and $65^{\circ} \mathrm{C}$ for $15 \mathrm{~min}$ ) using a thermal cycler (Hema 9600, Applied Biosystems). Real-time qPCR was performed using a Roche LC480 sequence detection system (Roche, Swiss). The following published primer sequences were used for the reactions [20]: PDE5, sense (5'- AAGCAAATGGTCACATTGGA - $3^{\prime}$ ) and antisense ( $5^{\prime}-$ TCTGGAAGTTCTGCACAAGG $\left.-3^{\prime}\right)$; GAPDH, sense ( $5^{\prime}$-CTGACTTCAACAGCGACACC- $\left.3^{\prime}\right)$ and antisense $\left(5^{\prime}\right.$-TGCTGTAGCCAAATTCGTTG- $\left.3^{\prime}\right)$. The PCR protocol used was as follows: denaturation at $95^{\circ} \mathrm{C}$ for $30 \mathrm{~s}$, followed by 40 cycles of annealing for $20 \mathrm{~s}$ each at $60^{\circ} \mathrm{C}$. Expression data were normalized to the geometric mean of a GAPDH housekeeping gene.

2.3. Western Blot Analysis. Four matched pairs of WDTC tumor tissues and adjacent nontumor tissues were harvested and lysed in $50 \mathrm{mM}$ Tris (pH 7.5), $100 \mathrm{mM} \mathrm{NaCl}$, $1 \mathrm{mM}$ EDTA, $0.5 \%$ NP40, $0.5 \%$ Triton X-100, $2.5 \mathrm{mM}$ sodium orthovanadate, $10 \mu \mathrm{M}$ of a protease inhibitor cocktail, and $1 \mathrm{mM}$ phenylmethylsulfonyl fluoride. Equal amounts of protein were electrophoretically separated on a 9\% SDS polyacrylamide gel and transferred to polyvinylidene fluoride membranes (Millipore, Bedford, MA, USA). The membranes were incubated at $4^{\circ} \mathrm{C}$ overnight with anti-human PDE5 rabbit monoclonal antibody (ab64179, 1:1000; Abcam Inc., Cambridge, USA). PDE5 expression was detected with horseradish peroxidase- (HRP-) conjugated goat anti-rabbit IgG secondary antibody $(1: 10,000$; Cell Signaling Technology, USA). The immunoreactive bands were visualized with an ECL chemiluminescence system (Tanon, Beijing, China). Anti- $\beta$-actin mouse monoclonal antibody $(1: 1000$; Cell Signaling Technology, USA) was used as a loading control.

2.4. Immunohistochemistry. Immunohistochemical staining was carried out on formalin-fixed, paraffin-embedded sections $(4 \mu \mathrm{m}$ thick) that were deparaffinized in xylene and rehydrated in an ethanol series with decreasing concentrations and rinsed in phosphate-buffered saline. Following this, antigen retrieval was performed with microwave treatment in $10 \mathrm{mM}$ citrate buffer ( $\mathrm{pH}$ 6.0). Immunohistochemical staining was carried out using the EnVision ${ }^{\mathrm{TM}}$ Kit (Dako, Hamburg, Denmark) according to the manufacturer's instructions. Endogenous peroxidase activity was quenched by treatment with $3 \%$ hydrogen peroxide for $15 \mathrm{~min}$. The sections were then incubated with primary anti-rabbit antibody against PDE5 (ab64179, 1:500) overnight at $4^{\circ} \mathrm{C}$. Then, the tissue sections were sequentially incubated with ready-to-use HRP immunoglobulin (EnVision Kit) for $30 \mathrm{~min}$ and developed with $3,3^{\prime}$-diaminobenzidine as a chromogen substrate. The nuclei were counterstained with Meyer's hematoxylin.

The extent of PDE5 immunostaining was evaluated independently by two pathologists who were blinded to the survival outcomes of the participants. They determined the proportion of positively stained tumor cells (staining area) and the intensity of staining. Staining intensity was scored as follows: 0, no staining; 1, weak staining (light yellow color); 2, moderate staining (yellow brown color); and 3, strong staining (brown color). The proportion of positively stained tumor cells was scored as follows: 0 , no positively stained tumor cells; $1,<5 \%$ positively stained tumor cells; $2,6-25 \%$ positively stained tumor cells; $3,26-50 \%$ positively stained tumor cells; and $4,>50 \%$ positively stained tumor cells. A modified immunoreactivity scoring method was used to evaluate the immunostaining results by multiplying staining intensity with the staining area (staining index, SI) 


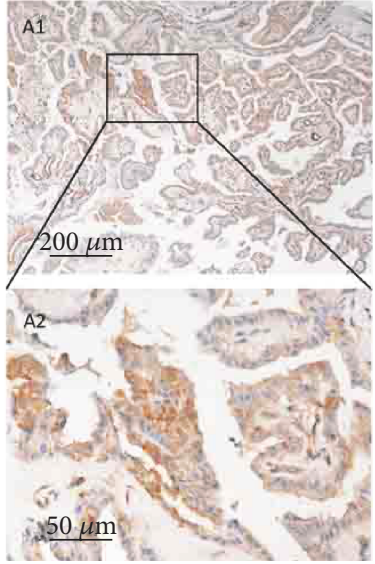

(a)

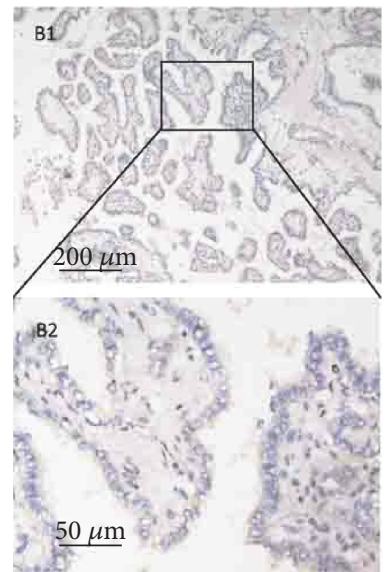

(b)

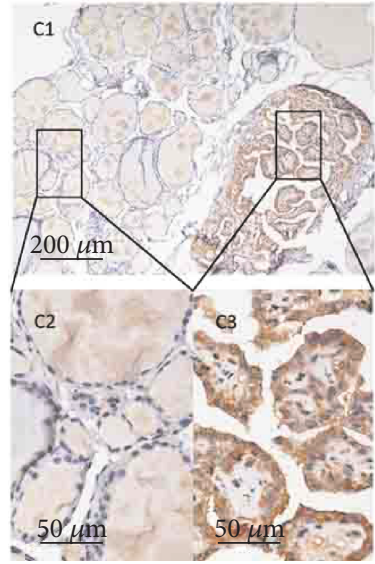

(c)

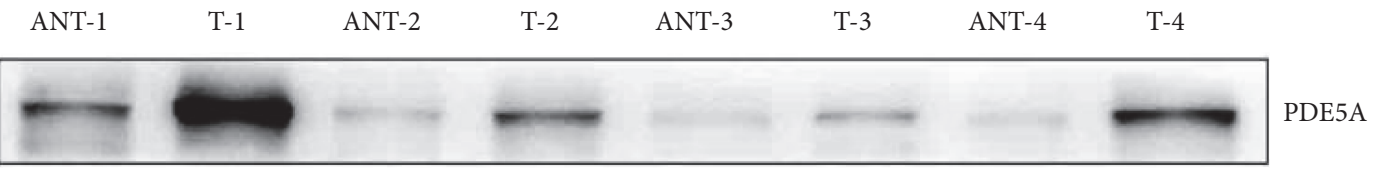

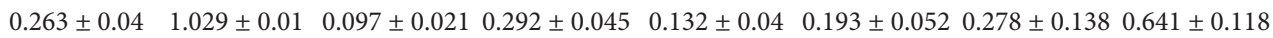

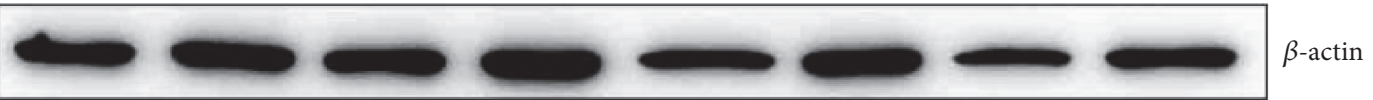

(d)

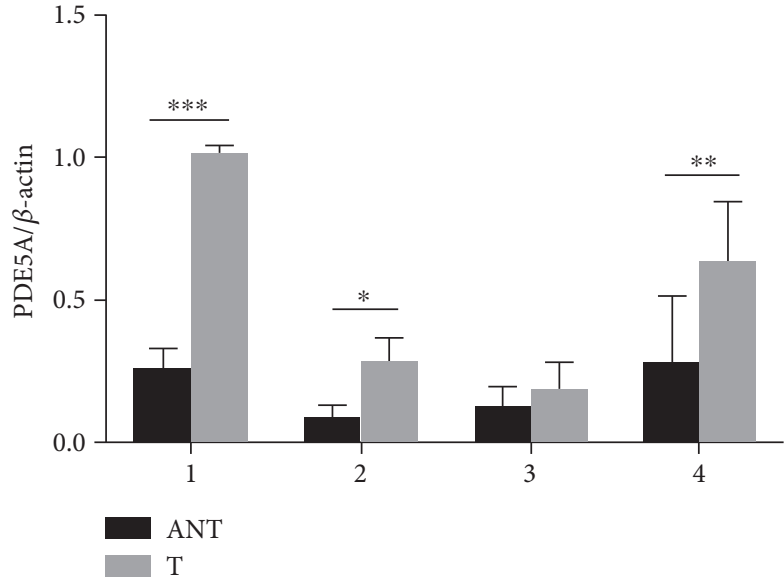

(e)

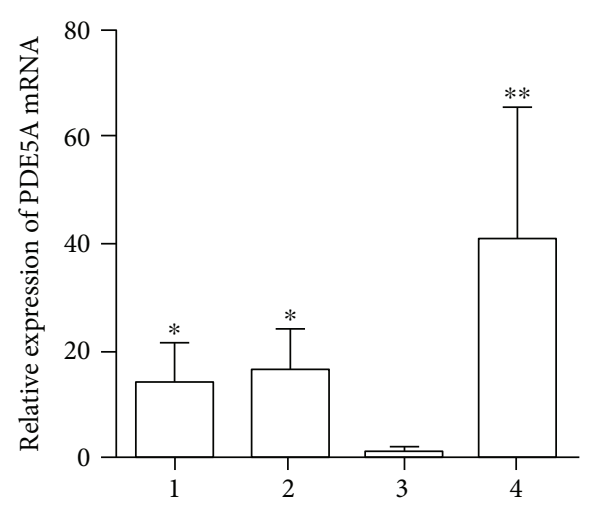

(f)

FIGURE 1: Upregulation of PDE5 expression in well-differentiated thyroid carcinoma (WDTC). (a, b, c) Expression of PDE5 in WDTCs as determined by immunohistochemistry. Strong staining $(\mathrm{A} 1, \times 10 ; \mathrm{A} 2, \times 40)$ and negative staining $(\mathrm{B} 1, \times 10 ; \mathrm{B} 2, \times 40)$ can be observed. A WDTC sample $(\mathrm{C} 1, \times 10)$ with strong PDE5 staining in the carcinoma tissues $(\mathrm{C} 2, \times 40)$ and normal thyroid follicular epithelial cells with weak staining $(\mathrm{C} 3, \times 40)$. ( $\mathrm{d}$ and e) Western blots show the expression levels of the PDE5 protein in four WDTC tumor tissues (T) and their paired adjacent noncancerous tissues (ANT); $\beta$-Actin was used as the loading control. (f) Real-time qPCR analysis results show the expression levels of PDE5 mRNA in WDTC tumor tissues relative to their paired adjacent noncancerous tissues (T/ANT). Values are given as the ratio of PDE5 expression to GAPDH expression $\left({ }^{*} P<0.05,{ }^{* *} P<0.01\right.$, and $\left.{ }^{* * *} P<0.001\right)$.

as previously described [20]. The PDE5 expression level in breast carcinoma lesions was determined by the SI, which was assigned values of $0,1,2,3,4,6,9$, or 12 . The optimal cutoff value was identified as follows: An SI score of $>6$ was considered to indicate high PDE5 expression, while an SI score of $\leq 6$ was considered to indicate low PDE5 expression.
2.5. Statistical Analysis. Chi-square test and logistic regression analysis were used for analysis of differences between the groups. Differences were considered significant when the $P$ value was $\leq 0.05$. SPSS v.23 was used for statistical analysis.

All cases were risk stratified on the basis of the clinical and pathological features in accordance with the 2015 American Thyroid Association Risk Stratification System 
TABLE 1: Clinicopathological features of 4 thyroid papillary carcinoma cases.

\begin{tabular}{lcccc}
\hline & Age & Tumor size & Lymph node status & Distant metastasis status \\
\hline Case 1 & 30 & $2 \mathrm{~cm}$ & Positive & Negative \\
Case 2 & 5 & $4 \mathrm{~cm}$ & Positive & Negative \\
Case 3 & 43 & $1.5 \mathrm{~cm}$ & Negative & Negative \\
Case 4 & 54 & Multiple & Positive & Negative \\
\hline
\end{tabular}

TABLE 2: Correlation between PDE5 expression and clinicopathological features.

\begin{tabular}{|c|c|c|c|c|c|}
\hline \multirow{2}{*}{ Clinicopathological parameters } & & \multirow{2}{*}{ Total } & \multicolumn{2}{|c|}{ The level of PDE5 expression } & \multirow{2}{*}{$P$ value } \\
\hline & & & High (\%) & Low (\%) & \\
\hline \multirow{2}{*}{ Gender } & Male & 36 & $19(36.5)$ & $17(33.3)$ & \multirow{2}{*}{0.733} \\
\hline & Female & 67 & $33(63.5)$ & $34(66.7)$ & \\
\hline \multirow{2}{*}{ Age (years) } & $<45$ & 67 & $39(75.0)$ & $28(54.9)$ & \multirow{2}{*}{0.032} \\
\hline & $\geq 45$ & 36 & $13(25.0)$ & $23(45.1)$ & \\
\hline \multirow{2}{*}{ Histological type } & PTC & 100 & $51(98.1)$ & $49(96.1)$ & \multirow{2}{*}{0.618} \\
\hline & FTC & 3 & $1(1.9)$ & $2(3.9)$ & \\
\hline \multirow{2}{*}{ Focus } & Single & 58 & $30(57.7)$ & $28(54.9)$ & \multirow{2}{*}{0.774} \\
\hline & Multiple & 43 & $21(42.3)$ & $22(45.1)$ & \\
\hline \multirow{2}{*}{ Invasion of capsule or ETE } & No & 89 & $45(86.5)$ & $44(86.3)$ & \multirow{2}{*}{0.969} \\
\hline & Yes & 14 & $7(13.5)$ & $7(13.7)$ & \\
\hline \multirow{2}{*}{ Distant metastasis } & M0 & 84 & $47(90.4)$ & $37(72.5)$ & \multirow{2}{*}{0.020} \\
\hline & M1 & 19 & $5(9.6)$ & $14(27.5)$ & \\
\hline \multirow{2}{*}{ LN involvement } & No & 46 & $16(30.8)$ & $30(58.8)$ & 0.004 \\
\hline & $\mathrm{N} 1$ & 57 & $36(69.2)$ & $21(41.2)$ & $\mathrm{OR}=2.79$ \\
\hline
\end{tabular}

ETE: extracapsular extension; PTC: papillary thyroid cancer; FTC: follicular thyroid cancer.

Guidelines, TNM staging, GAMES, and Mayo Clinic Scoring System (metastases, age, complete resection, invasion, size, or MACIS).

\section{Results}

3.1. Upregulation of PDE5 Expression in WDTC. Real-time qPCR and Western blot analysis showed that the expression levels of PDE5 mRNA and protein, respectively, were markedly higher in all four WDTC lesions than in their paired adjacent noncancerous tissues (Figures 1(d), 1(e), and 1(f)). All four cases were female, and their histological types were thyroid papillary carcinoma confirmed by pathology. The detail clinicopathological features were described in Table 1.

Furthermore, the immunohistochemical staining patterns revealed that PDE5 was mainly localized in the cytoplasm of the tumor cells. No staining or weak cytoplasmic staining was detectable in the follicular epithelial cells of the adjacent noncancerous thyroid tissue (Figure 1(c)).

3.2. Relationship between PDE5 Expression and the Clinicopathologic Features of WDTC. The immunohistochemical staining results showed that $52(50.5 \%)$ of the 103 WDTC patients had a high level of PDE5 expression $(\mathrm{SI}>6)$, whereas $51(49.5 \%)$ had a low level of PDE5 expression $(\mathrm{SI} \leq 6)$.
TABLE 3: PDE5 expression and lymph node involvement in patients aged $\geq 45$ years.

\begin{tabular}{lccc}
\hline \multirow{2}{*}{ LN involvement } & \multicolumn{2}{c}{ The level of PDE5 } & \\
& expression & P value \\
& High (\%) & Low (\%) & \\
\hline N0 & $1(7.7)$ & $13(56.5)$ & $\mathbf{0 . 0 0 5}$ \\
N1 & $12(92.3)$ & $10(43.5)$ & OR $=15.60$ \\
\hline
\end{tabular}

The correlation between PDE5 protein expression and the clinicopathologic features of WDTC is shown in Table 2. PDE5 expression was significantly correlated with age $(P=0.032)$, the regional lymph node status $(P=0.004)$, and the presence of distant metastasis $(P=0.020)$, according to the results of the chi-square $\left(\chi^{2}\right)$ test. We observed more significant differences in lymph node metastasis in patients who were over 45 years old $(\mathrm{OR}=15.60, P \leq 0.05)$ (Table 3).

\section{Discussion}

This study found a significant relationship between PDE5 expression and lymph node involvement in WDTC. PDE5 may be a potential marker of regional lymph node involvement and disease prognosis in WDTC. The results would be valuable from the viewpoint of future diagnosis. 
At variance with cAMP and its pathway, widely investigated as the main mediator of the TSH effects on thyroid cells, previous studies are widely focused on the TSH effect regulation of cAMP and its pathway on thyroid cells and involved in several thyroid diseases [21]. The role of the regulation of cGMP, mainly exerted by PDEs, in thyroid cancer is unclear. Sponziello et al. [18] found higher expression levels of PDE5A in tumors versus normal tissues in a group of 86 PTCs and noted the presence of higher expression levels of PDE in PTCs with BRAF V600E mutation. In PTCs, activating BRAF mutation represents the most frequent genetic alteration and is also considered as a marker of aggressiveness [22-25]. However, the significance of PDE5 expression with clinicopathologic features in these PTCs was unobserved. We confirmed that PDE5 expression was upregulated in WDTC tissues at both the mRNA and protein level compared to adjacent nontumor tissues. Further, our results revealed that overexpression of PDE5 in WDTC was significantly correlated with age, lymph node metastases, and distant metastases in a larger group of 103 WDTCs.

The role of PDE5 in cancer pathogenesis and prognosis is uncertain and complex. Peak et al. [26] concluded that PDE5 inhibition may help decrease the risk of developing colorectal and breast cancers, leukemia, and myeloma. PDE5 expression was found to have significantly higher levels in malignant breast tumors and had positive correlation with tumor grade, stage, and lymph node involvement [27]. Through cGMP/PKG-dependent neoplastic pathway, inhibition of PDE5 can induce apoptosis and prevent tumorigenesis in colon and breast tumor cells [28-30]. However, conflicting data suggest that inhibition of the NO/cGMP pathway can increase cellular motility, invasiveness, and cancer progression in breast tumor cells [31]. Previous study found that PDE5 inhibitors can block the proliferation of thyroid cancer cells in culture [18]. Further studies in vivo and vitro are needed to explain the mechanism of why PDE5 overexpression can be a driver of tumorigenesis and clarify whether specific inhibition of PDE5 may be proposed for the treatment of these tumors.

Cervical lymph node metastasis has been reported to be present in $20-90 \%$ of patients with WDTC $[8,16,32-34]$. The risk of regional recurrence is higher in patients with lymph node metastases, especially in those with more than 10 involved nodes and extracapsular extension [34]. The presence of lymph node metastasis is also indicative of a poorer prognosis [17]. Because regional lymph node metastasis is a significant indicator of disease prognosis or recurrence in clinical practice, it is important to determine whether regional lymph node metastasis is involved before surgery. In the present study, we observed high PDE5 expression in patients positive for regional lymph node metastases. Therefore, PDE5 may have potential for the identification of patients in whom aggressive treatment, such as prophylactic central lymph node dissection, would be beneficial. Since these results are only preliminary, tissue samples from more cases of WDTC may be required to confirm the significance of PDE5 in lymph node metastases.

\section{Conclusions}

In summary, our study investigated PDE5 expression in WDTC. We found high PDE5 expression in patients with lymph node metastasis; moreover, lymph node metastasis was associated with disease recurrence, treatment failure, and morbidity. Thus, the PDE5 expression level may in the future help identify patients who are required of aggressive treatment.

\section{Conflicts of Interest}

The authors declare that they have no potential conflicts of interest.

\section{Authors' Contributions}

Ning Zhang and Zeng Fang contributed in conducting immunohistochemistry staining, clinical data collection, and statistical analysis. Qiufang $\mathrm{Li}$ and Kebing Wang contributed in conducting Western blot and real-time qPCR. Zeng Fang and Songqi Li contributed in collecting the fresh tissue samples. Wen Li and Shenming Wang supervised the project and carried out experimental design and preparation of the final manuscript. All authors read and approved the final manuscript. Ning Zhang and Zeng Fang contributed equally to this article.

\section{Acknowledgments}

This study was supported by grants from the National Natural Science Foundation of China (81172337, 81370368, and 81672417).

\section{References}

[1] J. A. Sipos and E. L. Mazzaferri, "Thyroid cancer epidemiology and prognostic variables," Clinical Oncology, vol. 22, no. 6, pp. 395-404, 2010.

[2] R. M. Tuttle and R. Leboeuf, "Follow up approaches in thyroid cancer: a risk adapted paradigm," Endocrinology and Metabolism Clinics of North America, vol. 37, no. 2, pp. 419-435, 2008.

[3] T. Rago, P. Vitti, L. Chiovato et al., "Role of conventional ultrasonography and color flow-doppler sonography in predicting malignancy in 'cold' thyroid nodules," European Journal of Endocrinology, vol. 138, no. 1, pp. 41-46, 1998.

[4] F. D. Gilliland, W. C. Hunt, D. M. Morris, and C. R. Key, "Prognostic factors for thyroid carcinoma. A populationbased study of 15698 cases from the surveillance, epidemiology and results (SEER) program 1973-1991," Cancer, vol. 79, no. 3, pp. 564-573, 1997.

[5] A. R. Shaha, J. P. Shah, and T. R. Loree, "Patterns of nodal and distant metastasis based on histologic varieties in differentiated carcinoma of the thyroid," American Journal of Surgery, vol. 172, no. 6, pp. 692-694, 1996.

[6] J. L. Roh, J. Y. Park, and C. I. Park, "Total thyroidectomy plus neck dissection in differentiated papillary thyroid carcinoma patients: pattern of nodal metastasis, morbidity, recurrence, and postoperative levels of serum parathyroid hormone," Annals of Surgery, vol. 245, no. 4, pp. 604-610, 2007. 
[7] F. Randolph, Q. Y. Duh, K. S. Heller et al., "The prognostic significance of nodal metastases from papillary thyroid carcinoma can be stratified based on the size and number of metastatic lymph nodes, as well as the presence of extranodal extension at a surgical affairs committee's taskforce on thyroid cancer nodal surgery," Thyroid, vol. 22, no. 11, pp. 1144-1152, 2012.

[8] C. J. Hughes, A. R. Shaha, J. P. Shah, and T. R. Loree, "Impact of lymph node metastasis in differentiated carcinoma of the thyroid: a matched-pair analysis," Head \& Neck, vol. 18, no. 2, pp. 127-132, 1996.

[9] J. A. Beavo, "Cyclic nucleotide phosphodiesterase: functional implications of multiple isoforms," Physiological Reviews, vol. 75, no. 4, pp. 725-748, 1995.

[10] I. Levy, A. Horvath, M. Azevedo, R. B. de Alexandre, and C. A. Stratakis, "Phosphodiesterase function and endocrine cells: links to human disease and roles in tumor development and treatment," Current Opinion in Pharmacology, vol. 11, no. 6, pp. 689-697, 2011.

[11] E. T. Goluboff, A. Shabsigh, J. A. Saidi et al., "Exisulind (sulindac sulfone) suppresses growth of human prostate cancer in a nude mouse xenograft model by increasing apoptosis," Urology, vol. 53, no. 2, pp. 440-445, 1999.

[12] C. M. Whitehead, K. A. Earle, J. Fetter et al., "Exisulindinduced apoptosis in a non-small cell lung cancer orthotopic lung tumor model augments docetaxel treatment and contributes to increased survival," Molecular Cancer Therapeutics, vol. 2, no. 5, pp. 479-488, 2003.

[13] H. J. Thompson, C. Jiang, J. Lu et al., "Sulfone metabolite of sulindac inhibits mammary carcinogenesis," Cancer Research, vol. 57, no. 2, pp. 267-271, 1997.

[14] A. Das, D. Durrant, F. N. Salloum, L. Xi, and R. C. Kukreja, "PDE5 inhibitors as therapeutics for heart disease, diabetes and cancer," Phamacology \& Therapeutics, vol. 147, pp. 1221, 2015

[15] N. Passon, E. Bregant, M. Sponziello et al., "Somatic amplifications and deletions in genome of papillary thyroid carcinomas," Endocrine, vol. 50, no. 2, pp. 453-464, 2015.

[16] Z. Liu, P. Hou, M. Ji et al., "Highly prevalent genetic alterations in receptor tyrosine kinases and phosphatidylinositol 3kinase/akt and mitogen-activated protein kinase pathways in anaplastic and follicular thyroid cancers," The Journal of Clinical Endocrinology and Metabolism, vol. 93, no. 8, pp. 3106-3116, 2008.

[17] X. Mingzhao, "Molecular pathogenesis and mechanisms of thyroid cancer," Nature, vol. 3, pp. 184-199, 2013.

[18] M. Sponziello, A. Verrienti, F. Rosignolo et al., "PDE5 expression in human thyroid tumors and effects of PDE5 inhibitors on growth and migration of cancer cells," Endocrine, vol. 50, no. 2, pp. 434-441, 2015.

[19] L. J. Zhang, K. B. Wang, L. S. Liu et al., "Overexpression of GOLPH3 is associated with poor prognosis and clinical progression in pancreatic ductal adenocarcinoma," BMC Cancer, vol. 14, p. 571, 2014.

[20] W. Li, C. P. Yu, J. T. Xia et al., "Sphingosine kinase 1 is associated with gastric cancer progression and poor survival of patients," Clinical Cancer Research, vol. 15, no. 4, pp. 1393-1399, 2009.

[21] D. Russo, F. Arturi, E. Chiefari, and S. Filetti, "Molecular insights into TSH receptor abnormality and thyroid disease,"
Journal of Endocrinological Investigation, vol. 20, no. 1, pp. 36-47, 1997.

[22] E. Puxeddu, C. Durante, N. Avenia, S. Filetti, and D. Russo, "Clinical implication of BRAF mutation in thyroid carcinoma," Trends in Endocrinology and Metabolism, vol. 19, no. 4, pp. 138-145, 2008.

[23] L. M. Caronia, J. E. Phay, and M. H. Shah, "Role of BRAF in thyroid oncogenesis," Clinical Cancer Research, vol. 17, no. 24, pp. 7511-7517, 2011.

[24] T. Kunavisarut, "Diagnostic biomarkers of differentiated thyroid cancer," Endocrine, vol. 44, no. 3, pp. 616-622, 2013.

[25] E. Puxeddu and S. Filetti, "BRAF mutation assessment in papillary thyroid cancer: are we ready to use it in clinical practice?," Endocrine, vol. 45, no. 3, pp. 341-343, 2014.

[26] T. C. Peak, A. Richman, S. Gur, F. A. Yafi, and W. J. Hellstrom, "The role of PDE5 inhibitors and the NO/cGMP pathway in cancer," Sexual Medicine Reviews, vol. 4, no. 1, pp. 74-84, 2016.

[27] F. Karami-Tehrani, M. Moeinifard, M. Aghaei, and M. Atri, "Evaluation of PDE5 and PDE9 expression in benign and malignant breast tumors," Archives of Medical Research, vol. 43, no. 6, p. 470, 2012.

[28] R. Wang, I. K. Kwon, N. Singh et al., "Type 2 cGMPdependent protein kinase regulates homeostasis by blocking c-Jun N-terminal kinase in the colon epithelium," Cell Death and Differentiation, vol. 21, no. 3, p. 427, 2014.

[29] H. N. Tinsley, B. D. Gary, A. B. Keeton et al., "Sulindac sulfide selectively inhibits growth and induces apoptosis of human breast tumor cells by phosphodiesterase 5 inhibition, elevation of cyclic GMP, and activation of protein kinase G," Molecular Cancer Therapeutics, vol. 8, no. 12, p. 3331, 2009.

[30] T. Lan, Y. Chen, J. Sang et al., "Type II cGMP-dependent protein kinase inhibits EGF-induced MAPK/JNK signal transduction in breast cancer cells," Oncology Reports, vol. 27, no. 6, p. 2039, 2012.

[31] L. C. Jadeski, C. Chakraborty, and P. K. Lala, "Nitric oxidemediated promotion of mammary tumour cell migration requires sequential activation of nitric oxide synthase, guanylate cyclase and mitogen-activated protein kinase," International Journal of Cancer, vol. 106, no. 4, pp. 496-504, 2003.

[32] C. S. Grant, "Recurrence of papillary thyroid cancer after optimized surgery," Gland Surgery, vol. 4, no. 1, pp. 52-62, 2015.

[33] B. Cady, C. E. Sedgwick, W. A. Meissner, J. R. Bookwalter, V. Romagosa, and J. Werber, "Changing clinical, pathologic, therapeutic, and survival patterns in differentiated thyroid carcinoma," Annals of Surgery, vol. 184, no. 5, pp. 541-553, 1976.

[34] I. Levy, A. Horvath, M. Azevedo, R. B. de Alexandre, and C. A. Stratakis, "Phosphodiesterase function and endocrine cells: links to human disease and roles in tumor development and treatment," Current Opinion Parmacology, vol. 11, no. 6, pp. 689-697, 2011. 


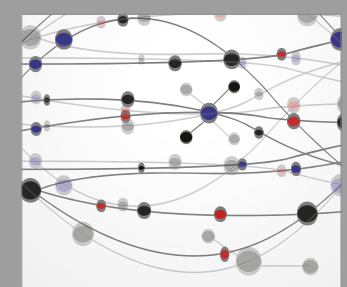

The Scientific World Journal
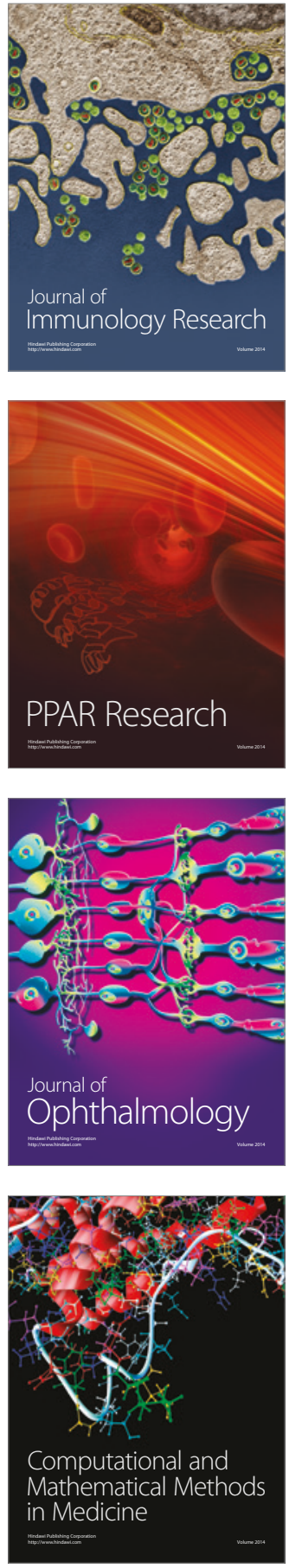

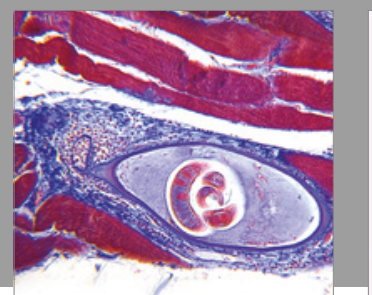

Gastroenterology Research and Practice
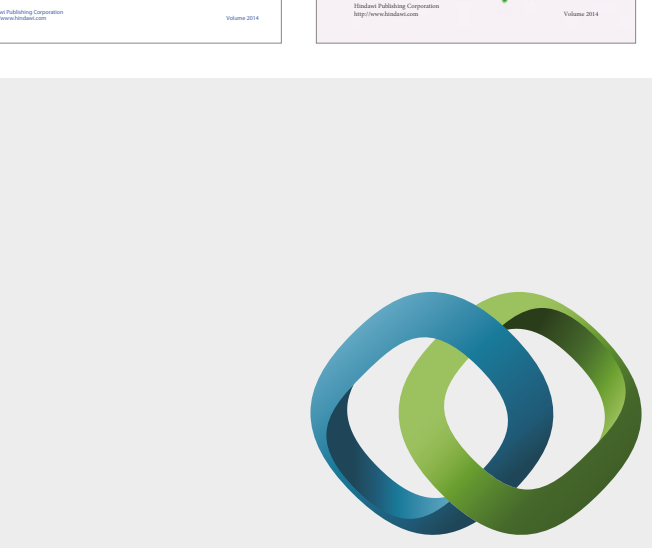

\section{Hindawi}

Submit your manuscripts at

https://www.hindawi.com
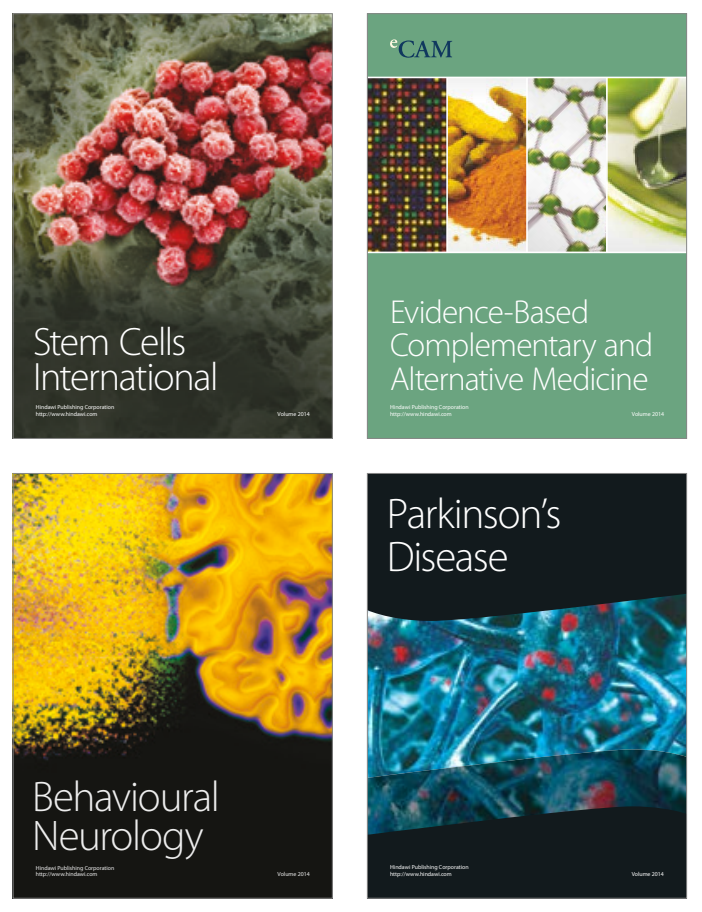
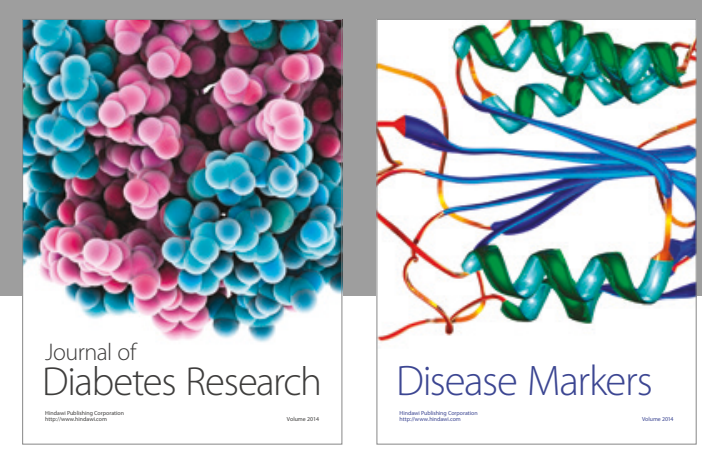

Disease Markers
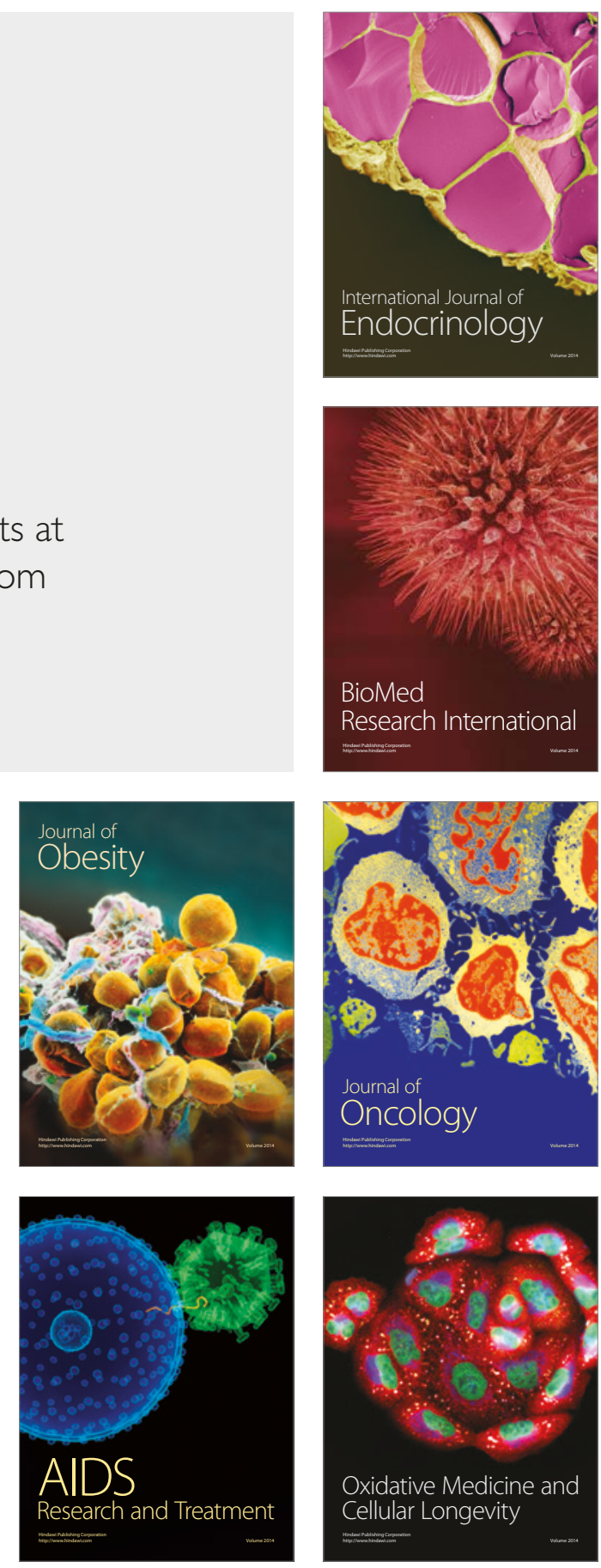